\title{
When Compliance Checks are Just the Start of the Journey: an Aviation Case
}

\author{
Mart de Vré 1,* $^{*}$ and Delia A. Richardson ${ }^{1}$ \\ ${ }^{1}$ Aviation Academy, Amsterdam University of Applied Sciences, The Netherlands
}

\begin{abstract}
Compliance with standards is assessed through internal and external audits, the findings of which are viewed as imperfections to be quickly repaired. A zero-findings mentality underlies companies that want to excel before the eyes of the authorities, customers, insurance companies and competitors. However, scholars and professionals over the last decades agree that compliance is a necessary but not sufficient condition for optimum system performance. The current study was initiated by a Ground Service Provider that in 2017 underwent an IATA Safety Audit for Ground Operations and revealed several findings, especially in the documentation, such as missing parts, non-compliant material and lack of detail. Their goal was to pass the next audit without any findings within the documented procedures of six operational departments. To assess the audit documentation criteria, the researchers visited the operational departments, analysed 186 procedures, and conducted six semi-structured interviews with managers/supervisors and nine interviews with operational personnel. The analysis showed that all documents were properly controlled, but four departments had duplicated generic guidance material from $6 \%$ to $83 \%$ of the text checked. The interviewees claimed that understandability was not optimum due to language barriers and the non-tailoring of the content to their needs, leading to some staff disregarding manuals and consulting their supervisor instead. Other remarks included the long length of the documents, lack of knowledge of how to access online material, ignorance of the existence of documentation access points, and low technical accuracy. Overall, the results suggested room for improvement. Most importantly, through this research, the specific Ground Service Provider gained a better understanding behind non-compliance and had the opportunity to improve the quality and communication of its procedures. This study showed that even when compliance is the target, substantial improvement moves beyond box-ticking and engages employees in the revelation and mitigation of system imperfections.
\end{abstract}

Keywords: Compliance; Zero-findings; Ground Service Provider; Procedures; Documentation.

\section{INTRODUCTION}

Internal and external audits tend to be the path through which companies seek compliance with standards and ensure the application of best practices. Findings from audits are viewed as imperfections to be fixed as soon as possible. Companies tend to want to go for zero-findings that suggest the achievement of full compliance with standards or application of best practice and a minimum level of quality (ICAO, 2016). A zero-findings mentality underlies companies that want to excel before the eyes of the authorities, customers, insurance companies and competitors. Compliance is a necessary but not sufficient condition for optimum system performance. Non-compliance provides a roadmap to what needs to be

\footnotetext{
*Corresponding author: +31(0)642537618, martdevre@gmail.com
} 
done to ensure compliance. However, compliance is not an indication or guarantee of quality standards (CANSO, 2011).

In 2007, the IATA Safety Audit for Ground Operations (ISAGO) made its entrance in the aviation industry as a way to improve safety and enhance operations for Ground Service Providers (GSP). ISAGO is based on industry-proven quality audit principles and is structured to ensure a standardized audit with consistent results across aviation businesses. The implementation of the ISAGO standards and recommended practices aim to improve safety as well as manage airline costs by reducing ground accidents and injuries. According to IATA, the benefits of ISAGO are increased safety operations/oversight, process efficiency, quality standards and understanding of high-risk areas. When GSPs are compliant with the standards of ISAGO, the companies should notice a reduction of ground hazards affecting flight safety, aircraft ground damage, personnel injury, redundant audits and costs (Suidan, 2010).

To achieve ISAGO certification, GSPs must comply with ISAGO's Standards and Recommended Practices (GOSARPs) and prove their compliance every two years to the ISAGO auditors by presenting their documentation and implementation of the GOSARPs. The approximately 350 GOSARPs are described in the ISAGO Standards Manual and are divided into different operational sections which are Organization and Management (ORM) Cargo, Load Control (LOD), Passenger and Baggage Handling (PAB), Aircraft Handling and Loading (HDL), Aircraft Ground Movement (AGM), and Cargo and Mail Handling (CGM). The GOSARPs need to be implemented and documented throughout the operational and organizational departments of the GSP. GOSARPs must comprise controlled documents that are actively maintained and used in the organisation. Nonetheless, GOSARPs need to be established, activated, integrated, incorporated, deployed, installed, maintained and made available, as part of the operational system, and monitored and evaluated for continued effectiveness (IATA, 2017).

A successful audit rests on the full function of procedures that are documented and implemented in the operational and organizational environment. A successful audit is also determined by the collaboration and effort regarding the safety principles of ISAGO within all disciplines in the organization. When all these factors are executed properly, the ISAGO audit will have zero-findings. The results intend to reflect the company's functioning with the highest degree of safety, accuracy, and professionalism (IATA, 2017).

In 2017, the researched GSP was audited for compliance with ISAGO. The operational and organisational departments were inspected to determine to what extent their documented and implemented procedures were compliant with the GOSARPs. After the audit, the GSP was proud to announce their compliance with ISAGO. However, this was without zero-findings. There were several non-conformances which subsequently needed to be addressed. One of the non-conformances was that operational procedures were not properly documented. Per department, the documented procedures were either missing, non-ISAGO, copied from the IATA Ground Operations Manuals (IGOM) or incomplete. With the current non-conformant documentation, the GSP had the risk that the next ISAGO audit will again lead to findings. As the researched company wants to excel in the eyes of the authorities, customers, insurance companies and competitors, this is not in line with the GSP's values.

To deal with this problem and provide solutions for improvement, the gap between the procedures that are already documented and the procedures that need to be documented was investigated. Consequently, corrective actions were provided to pass the next ISAGO audit with zero-findings. Thus, this research aimed to determine how the GSP could close the gaps between the current and preferred situation without any findings within the documented procedures and the operational departments. The research focused on the GSP's operational environment that performs the following activities: Cargo Export, Special Cargo Handling, Cargo Import, Passenger Handling, Ramp Handling, and Mail Handling. This study did not focus on the implementation part of ISAGO.

The rest of this paper is as follows. Section 2 discusses the literature underpinning the research. Section 3 outlines the methodology used for this research. Section 4 presents and 
analyses the results respectively. Section 5 discusses the results, and section 6 gives a conclusion and discloses the recommendations for the GSP its operational departments regarding their compliance of documentation with ISAGO and its GOSARPs.

\section{LITERATURE REVIEW}

\subsection{ISAGO Assessment of Documentation}

One tool that ISAGO auditors use to determine compliance is the ISAGO Standards Manual. The manual describes GOSARPs and provides guidance for the auditor to evaluate documentation and subsequent implementation. The guidance includes the minimum requirements for documentation and implementation to be found in the GSP's documentation and operations. To be compliant with the GOSARPs, the GSP must understand the GOSARP's intent, instruction and recommendation, and document the operations accordingly (IATA, 2017).

Moving beyond the compliance with the specification of the GOSARP, IATA's Airport Handling Manual (AHM) (2017) provides the assessment of documentation based on the total conformance of the program. To assess conformity with a GOSARP as documented, an auditor must be able to find the applicable ISAGO specifications published in a Controlled Document, such as a manual, handbook or other similar publications, that contains content approved by the provider. The minimum document control techniques must be applied: identification reference, edition and/or revision number, edition and/or revision date. A Controlled Document must also have an associated process for revision and distribution that ensures appropriate operational personnel always have access to the current version of the document. However, additional criteria to assess conformity with documented GOSARPs are (IATA AHM, 2017):

1. Both paper and electronic forms of documentation are acceptable as long as the criteria for a Controlled Document are met.

2. The content of a document must be written in a style and format that can be understood by operational personnel, and, clearly and accurately represents the meaning and intent of the specification.

3. Secondary documents such as meeting minutes, bulletins, records, checklists, etc., cannot usually be used as primary documentation references unless the document has references that link to provider manuals, procedures, and policies which relate directly to that GOSARP requirement.

4. Documents of a temporary or transitory nature, are only acceptable as primary sources of Controlled Documentation when the information has been reproduced and included in the content of a Controlled Document.

5. There shall be proof of both distribution and receipt of documentation to all relevant personnel.

6. The provider can use its own operational manual or implement IATA Ground Operations Manual (IGOM) which includes industry agreed and standardized procedures for ground operations.

\subsection{Procedure Quality, Compliance and Development}

The challenge with documented procedures is the way in which they are presented to their targeted users. Having documentation available or being compliant with applicable regulations is the first step. The next critical step is to ensure that the documentation will be used actively as a tool to enhance operations. Moreover, documentation needs to be understandable and seen as effective by its users. Several researchers expressed the importance of having proper procedural documentation and what it takes to achieve 
understandable and effective documentation. Peterson (2006) refers to the importance of operating procedures and claims that effective written procedures should be at least properly designed, effectively distributed and tested for optimal effectiveness (Peterson, 2006). Peterson (2006) also argues that effective written procedures should all have a consistent format that includes how-to information.

A framework that can be used to test the overall quality of documentation was suggested by Schiesser (2002). The framework has ten criteria "...to show to what degree the material is suitable for use..." (p. 1). The ten criteria consist of ownership, readability, accuracy, thoroughness, format, accessibility, currency, ease of update, effectiveness, and accountability (Schiesser, 2002).

Regarding understandability, the challenge companies face is that they consist of people from many backgrounds, cultures, and languages (Henman, 2017). For example, operational personnel who are not native English speakers, their literacy level may be lower than anticipated, but also depends on training, educational level and experience. In addition, translation between languages is difficult to manage, as words and sentences in different languages are not always identical to the meaning and function of the native language. The challenge regarding the involvement of multiple cultures in an organization is that symbols and concepts can be interpreted differently per culture. As a result, ensuring a unified understanding of documentation can be very difficult (Barbé et al., 2016).

Fortunately, there are some solutions to this problem. "Graphics, animation, sound, and simulation can be employed to illustrate proper procedures and processes, rather than merely explain them. Skilful use of these instructional techniques can engage employees and enhance comprehension, ensuring that the procedure's intent is achieved" (Peterson, 2006, p. 3). Beluško et al. (2016) also support this assertion, describing that the picture-based procedures/photographic work instructions are the most efficient format for documentation, and, certainly, more efficient than textual instructions. Beluško et al. (2016) also present the learning pyramid with percentages of information to be remembered after using a certain format of transferring knowledge (Figure 1). Through the learning pyramid, they showed that audio-visuals are twice as effective as textual-based knowledge transfer. However, demonstrations, discussion groups, learning by doing and teaching others, show larger information processing than the audio-visuals and reading texts.

To check the understanding and application of new documentation, Peterson (2006) suggests that it should be tested. Testing new documentation generates information to identify the effectivity of new documentation among personnel and possible inefficiencies caused by insufficient training. Additionally, to avoid confusion due to multiple revisions of documents within a short time frame, procedures need to be distributed in a timely manner and employees have to validate the receipt and understanding of the procedures.

To enhance employee engagement in the use of documentation, Amare (2012) claims that an effective procedure is written with the input of every employee involved in the process. Individuals who participate in the process are positive to generate ideas, are more acceptive of the documentation and feel empowered in it. This is opposite to the case when a procedure is imposed by management without the workforce's opinion and input. Whoever writes the procedure, the most important thing is to convey a clear instruction in a manner that is easy for everyone to understand (Amare, 2012). 


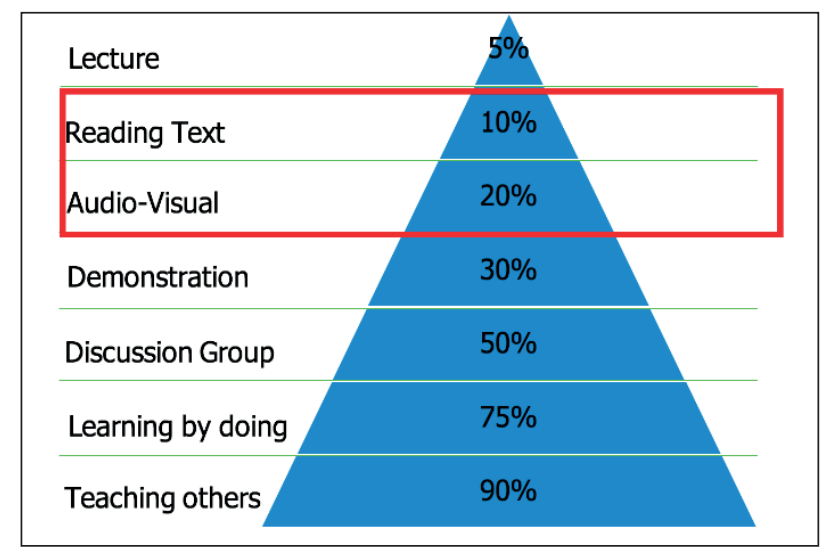

Figure 1: Information processing (Beluško et al., 2016)

\section{METHODOLOGY}

Data was gathered through desk research (i.e. documental analysis) and field research (i.e. semi-structured interviews) and assessed against the criteria necessary to achieve zerofindings, as presented in section 2.1 and section 2.2. Based on the requirements mentioned in section 2.1, the researchers determined the assessment criteria by analysing the objectives of the content. For example, the content of a document must be written in a style and format that can be understood by operational personnel, and, clearly and accurately represents the meaning and intent of the specification. In this example, the researchers extracted the criterion Format to measure the understandability and the accuracy of the described specification in the GSP's documentation. The researchers extracted five criteria from the requirements of ISAGO. These included: Access (1), Accuracy (2), Controlled Document (3), Distribution (4), and Format (5). The criteria are accompanied by the measurable indicators which were derived from the quality framework of Schiesser (2002). Although the framework described ten criteria to measure quality, due to time limitations, the researchers limited this research to the five criteria of the $\mathrm{AMH}$ paired with the measurable indicators of the quality framework.

The documentation was assessed according to the criteria which along with the indicators were validated by an ISAGO auditor who confirmed that they were sufficient to demonstrate compliance with ISAGO. Based on the indicators, the criteria were either assessed through field research, desk research or both.

The documentation criteria which were only used in field research are described in Table 1. Appropriate semi-structured interview questions were raised to gather data of the criteria Access (1), Accuracy (2), Distribution (4) and Format (5).

Table 1 Criteria and interview questions

\begin{tabular}{||l|l|l||}
\hline CRITERIA & \multicolumn{1}{|c|}{ INDICATORS } & \multicolumn{1}{c|}{ INTERVIEW QUESTIONS } \\
\hline Access (1) & $\begin{array}{l}\text { Ease of difficulty of } \\
\text { accessibility }\end{array}$ & $\begin{array}{l}\text { Via what channel can the work } \\
\text { instructions } \\
\text { and procedures be accessed? }\end{array}$ \\
\cline { 3 - 4 } & & $\begin{array}{l}\text { Are work instructions and procedures } \\
\text { easy to access? }\end{array}$ \\
& & $\begin{array}{l}\text { Why is it easy to access? Why is it hard } \\
\text { to access? }\end{array}$ \\
\hline Accuracy (2) & $\begin{array}{l}\text { Technical accuracy of of } \\
\text { documentation. }\end{array}$ & $\begin{array}{l}\text { Are the work instructions and procedures } \\
\text { representing the operational activities? If } \\
\text { not, why not? }\end{array}$ \\
\hline
\end{tabular}




\begin{tabular}{||c|l|l||}
\hline CRITERIA & \multicolumn{1}{|c|}{ INDICATORS } & \multicolumn{1}{c|}{ INTERVIEW QUESTIONS } \\
\hline Distribution (4) & $\begin{array}{l}\text { Communication of } \\
\text { revisions, accessibility } \\
\text { of revisions. }\end{array}$ & $\begin{array}{l}\text { How are revised work instructions and } \\
\text { procedures communicated towards you? } \\
\text { If not, why not? }\end{array}$ \\
\hline $\begin{array}{l}\text { Format (5) } \\
\text { (Understandability) }\end{array}$ & $\begin{array}{l}\text { Clarity and simplicity of } \\
\text { written documentation. }\end{array}$ & $\begin{array}{l}\text { Do you work according to the work } \\
\text { instructions? If not, why not? }\end{array}$ \\
& $\begin{array}{l}\text { Is the format style of the work instructions } \\
\text { and procedures understandable? If not, } \\
\text { why not? }\end{array}$ \\
& $\begin{array}{l}\text { Is the written style of the work instructions } \\
\text { and procedures understandable? If not, } \\
\text { why not? }\end{array}$ \\
\hline
\end{tabular}

According to the AHM (2017), operational personnel must be able to understand documentation. Therefore operational personnel and managers/ supervisors were interviewed on the operational processes. Within the operational departments, supervisors and operational personnel use the work instructions and procedures in their daily duties. For this research, five operational personnel and two supervisors from six departments (department 1 - department 6) were approached. However, during the research, only fifteen employees of the company responded to the interviews. Six semi-structured interviews with managers/supervisors and nine interviews with operational personnel were eventually carried out to collect information on the GSP's current situation regarding their compliance with ISAGO's documented procedures. Interviews were conducted until theoretical saturation was reached. The interviews were analysed to make sense of the data by finding similarities and differences between the interview data and the data from the documental analysis. The researchers started with open coding to categorize the data by colour followed by the axial coding technique used to identify linkages in the data and classify the similarities and differences in the data. The findings of the interviews were validated by the heads of each department.

The researchers also reviewed the 186 documents against the standards of the ISAGO Standards Manual to identify to what extent the documentation documented the respective GOSARPs and whether findings could be identified. The factors Accuracy (2), Controlled Document (3), and Format (5) were used to measure compliance with ISAGO; Table 2 shows the criteria with the appropriate measurable indicators.

Table 2 Criteria document research (IATA AHM, 2017)

\begin{tabular}{||c|l||}
\hline \hline CRITERIA & INDICATOR \\
\hline Accuracy (2) & $\begin{array}{l}\text { Technical accuracy, compliance with GOSARP as stated in the } \\
\text { ISAGO Standards Manual }\end{array}$ \\
\hline Controlled Document (3) & $\begin{array}{l}\text { Identification reference, Edition and/or revision number, } \\
\text { Edition and/or revision date. }\end{array}$ \\
\hline Format (5) & $\begin{array}{l}\text { Describing an actual process instead of duplicating from IATA } \\
\text { guidance materials (IGOM \& AHM) }\end{array}$ \\
\hline
\end{tabular}

The technical Accuracy of the documentation's content was reviewed against the approximately 350 processes and procedures of ISAGO's GOSARPs which are included in the ISAGO Standards Manual (2017). Controlled Document was assessed on the presence of an identification reference that refers to the procedure, Edition/revision number and date which means an updated version number and date of revision is available. The documents were also checked to detect whether their content was duplicated from the IGOM $6^{\text {th }}$ edition 
(2017) and AHM 37th edition (2017) or if the GSP had produced their own, customised content (Format).

\section{RESULTS}

\section{Criterion 1: Access}

According to ISAGO, appropriate operational personnel must always have access to the current version of the documentation. The results of the semi-structured interviews reveal that not all employees have access to the documentation in the operational environment. The operational personnel of departments 5 and 6 did not know that documentation is available. They solely relied on the instructions of senior personnel who do use and have the knowledge of and from the available documentation. The semi-structured interviews also revealed that some personnel in all six operational departments did not have access to the company's intranet where the documentation could be found. In addition, the interviewees expressed that the accessibility of documentation via the intranet was not optimal due to time pressure. The interviewees stated that they often had to work beyond boundaries to accomplish their daily tasks and little time remained actually to read documentation when needed. They also stated that in the cargo industry time pressure occurs due to swift changes in daily practices (e.g. order changes of customers, delays of aircraft). Moreover, the interviewees were unwilling to spend their limited time to access documentation; instead, information and instructions were shared amongst colleagues.

\section{Criterion 2: Accuracy}

The information from the semi-structured interviews revealed that the interviewees differed in their responses to the question of whether the work instructions and procedures were representing the operational activities. Several participants stated that they used documentation to perform operations, but others declared that they did not use documentation or they solely depended on the instructions of senior operational personnel or offered during training. Notably, only employees from the departments 5 and 6 were the ones who stated that they were working according to the instructions of senior personnel. Also, interviewees who were not using documentation to perform operations stated that they often had to deviate from the guidance of documentation due to time pressure during operations. It is expressed that the procedures do not align with the turbulent operations happening in the operative environment.

The 186 documents analysed against the respective GOSARPs showed findings across all six departments, mainly missing information in the documentation or not describing the process at all. For example, the department responsible for cargo export had a procedure which involves the handling of fragile cargo, but this was missing detailed information about the handling process of fragile cargo that it needs to be placed on top of regular cargo. Table 4 presents the documentation incompliant with ISAGO's GOSARPs.

Table 4 Findings in operational departments

\begin{tabular}{||l|l|l|l||}
\hline DEPARTMENT & GOSARP & FINDING & $\begin{array}{l}\text { LACKING IN } \\
\text { DOCUMENTATION }\end{array}$ \\
\hline \multirow{1}{*}{1} & CGM 1.3.6 & Missing information & $\begin{array}{l}\text { A process which describes that } \\
\text { "fragile cargo has to be on top of } \\
\text { regular cargo". }\end{array}$ \\
\cline { 2 - 4 } & CGM 2.2.2 & $\begin{array}{l}\text { Not described in } \\
\text { documentation }\end{array}$ & $\begin{array}{l}\text { A process of handling High-risk } \\
\text { cargo. }\end{array}$ \\
\hline
\end{tabular}




\begin{tabular}{|c|c|c|c|}
\hline DEPARTMENT & $\overline{\text { GOSARP }}$ & "FINDING & $\begin{array}{l}\text { LACKING IN } \\
\text { DOCUMENTATION }\end{array}$ \\
\hline 2 & CGM 1.3.3 & Missing information & $\begin{array}{l}\text { A process for the separation of } \\
\text { human remains cargo from } \\
\text { incompatible load, and there is } \\
\text { no list which indicates the } \\
\text { identification of incompatible } \\
\text { load. }\end{array}$ \\
\hline \multirow{3}{*}{3} & PAB 1.2.1 & Missing information & $\begin{array}{lll}\text { No seat } & \text { requirements } \\
\text { personnel. } & \end{array}$ \\
\hline & PAB 1.2.5 & $\begin{array}{l}\text { Not described in } \\
\text { documentation }\end{array}$ & $\begin{array}{l}\text { A process to prove oversight of } \\
\text { the scale controls and } \\
\text { calibration. }\end{array}$ \\
\hline & PAB 1.5.4 & Missing information & $\begin{array}{l}\text { A process that describes the } \\
\text { authorized and qualified person } \\
\text { for checking weapons and the } \\
\text { requirements of packaging and } \\
\text { labelling of weapons. }\end{array}$ \\
\hline 4 & HDL 2.3.4 & Missing information & $\begin{array}{l}\text { Indication that all applicable staff } \\
\text { is familiar with the applicable } \\
\text { handling labels. }\end{array}$ \\
\hline \multirow{3}{*}{5} & CGM 1.3.1 & $\begin{array}{l}\text { Not described in } \\
\text { documentation }\end{array}$ & $\begin{array}{l}\text { A process that describes the } \\
\text { handling and acceptance of live } \\
\text { animals. }\end{array}$ \\
\hline & CGM 1.3.4 & $\begin{array}{l}\text { Not described in } \\
\text { documentation }\end{array}$ & $\begin{array}{l}\text { A process to handle Valuable } \\
\text { Cargo. }\end{array}$ \\
\hline & CGM 1.3.10 & $\begin{array}{l}\text { Not described in } \\
\text { documentation }\end{array}$ & $\begin{array}{l}\text { A process to ensure any type of } \\
\text { special cargo shipment is broken } \\
\text { down, delivered or transferred to } \\
\text { the consignee. }\end{array}$ \\
\hline 6 & CGM 1.1.4 & Missing information & $\begin{array}{l}\text { How to calibrate a scale and } \\
\text { periodically check the accuracy } \\
\text { of the scale by using determined } \\
\text { weights. }\end{array}$ \\
\hline
\end{tabular}

\section{Criterion 3: Controlled Document}

Each of the 186 documented procedures and work instructions available on the company's intranet was reviewed against the three criteria: identification reference, edition and/or revision number, and edition and/or revision date. These are necessary to comply with the criterion Controlled Document (Table 2). Analysis showed that all documents of all six operational departments were compliant with the criterion Controlled Document.

\section{Criterion 4: Distribution}

One of the requirements of ISAGO is that there must be proof of distributing documentation towards relevant personnel. The analysis of the criterion Distribution showed diverse answers across the operational departments. Most interviewees notified that documentation and revisions had been distributed via memos and emails to the employees. However, not all operational departments acknowledged that there was proof of their personnel receiving the documentation. Nevertheless, department 3 and department 4 used a system to provide proof that the documentation was read and understood by asking 
personnel to sign a respective log. The participants from the other operational departments did not express that there was such a system to distribute documentation and provide proof that the documentation was read, received, and understood.

\section{Criterion 5: Format}

The criterion Format was analysed based on two requirements: (a) whether the procedures describe an actual process rather than duplicating IATA guidance materials, and (b) understandability of the documentation by all operational personnel. The researchers compared IATA's guidance materials IGOM $6^{\text {th }}$ edition and AHM $37^{\text {th }}$ edition with the operational department's documentation. The analysis revealed that four operational departments merely duplicated several documents from the IGOM $6^{\text {th }}$ edition and AHM $37^{\text {th }}$ edition. Two operational departments had not duplicated any documentation from the IGOM $6^{\text {th }}$ edition or AHM $37^{\text {th }}$ edition.

Table 3 shows the distribution of documents that had been duplicated across the six departments. It can be noticed that the extent of compliance deferred per department; department 3 had $86 \%$ of its documents duplicated, whereas department 4 had only $6 \%$ duplicated documents. The difference becomes even more salient when considering that department 3 only maintains seven documents, from which six were duplicated, and department 4 has 80 documents from which five were duplicated.

Table 3 Number of documents duplicated and non-duplicated documents

\begin{tabular}{||l|c|c||}
\hline DEPARTMENT & $\begin{array}{c}\text { NUMBER OF } \\
\text { DOCUMENTS }\end{array}$ & $\begin{array}{c}\text { NUMBER AND PERCENTAGE OF DUPLICATED } \\
\text { DOCUMENTS }\end{array}$ \\
\hline 1 & 30 & $8(27 \%)$ \\
\hline 2 & 41 & $5(12 \%)$ \\
\hline 3 & 7 & $6(86 \%)$ \\
\hline 4 & 80 & $5(6 \%)$ \\
\hline 5 & 8 & $0(0 \%)$ \\
\hline 6 & 55 & $0(0 \%)$ \\
\hline
\end{tabular}

The assessment of the second part of the Format criterion collected various answers from personnel in the operational departments. In general, the understandability of the current documentation differed within the operational departments. Some documents were understandable by operational personnel and other documents were not read at all by employees because of their composition and content. The interviewees in departments 5 and 6 expressed appreciation of their documentation as its Format represented and reflected the wishes of the personnel by including pictures and little text in the documents. However, the interviewees of the remaining departments stated that the documents were too long and contained too much text. Furthermore, some interviewees argued that the documentation did not suit the composition different active nationalities within their departments. The text was often written in difficult English or Dutch which was leading misunderstandings of the documentation. These factors combined with the high pressure of operations in the work environment reduced dramatically the willingness of personnel to read the documentation.

\section{DISCUSSION}

The analysis of the results concerning the compliance of the GSP's documentation with the standards of ISAGO showed that documentation was missing, non-ISAGO compliant, copied from the IGOM or incomplete. However, this study found that these constraints were not the only non-conformances within the documentation. The results of the data collection 
revealed that solely the criterion Controlled Document was aligned with the requirements of ISAGO; the criteria Access, Accuracy, Distribution, and Format were not fully met.

One of the constraints in the documentation assessed was that it was not adapted to the composition of the operational environment. According to ISAGO, the documentation must be understood by operational personnel. The results showed that it contained too much text for personnel to read. This was coupled with the fact that employees were often operating under time pressure and exposed to language barriers. Thus, the particular finding accords with the work of Beluško et al. (2016) who presented that textual work instructions are processed almost half as long as audio-visual work instructions. In addition, when it comes to remembering procedures, activating more senses will enhance remembering the procedures better. Therefore, it seems that procedures containing too much text are not effective and do not comply with the requirements of ISAGO as long texts are not understandable by operational personnel. Department 6 was an exemption in this situation; they had documentation containing pictures and little text and understandable by personnel.

Moreover, the results showed that the Access criterion was not fully met. The operational personnel of department 5 and department 6 did not have knowledge of any available documentation because senior personnel were the ones to use the documentation and give instructions to operational personnel. Additionally, numerous personnel who needed to access the documentation on the company's intranet cannot access the intranet platform as they claimed not to have the login data to access the documentation.

Furthermore, several procedures that should comply with the GOSARPs of ISAGO were missing in the documentation or were incomplete. An explanation of this could be that the documents that were duplicated from the IGOM $6^{\text {th }}$ edition provided a baseline on which a GSP needs to build their procedures. When duplicating the procedures from the IGOM $6^{\text {th }}$ edition, details that needed to be documented are not present and will result in not complying with the GOSARP according to ISAGO. It is also possible that the management of the operational departments decided to duplicate the documentation from the guidance materials to save time and money.

Finally, the overall results of this study naturally lead to the question of how the GSP had successfully completed the audit in 2017 under so many problems in the documentation. Audits tend to suggest that, once a company has completed the audit, it will deliver a certain level of quality, performance and safety. However, in this case, there were a lot of things that needed to be fixed. So why did the particular GSP pass the audit in the previous instance? Did this GSP hide a few of its inefficiencies from the auditors, or were the auditors not vigilant enough when carrying out the auditing tasks? Having detected plenty of problems in the documentation of the specific GSP, we can claim that a successful audit does not directly mean high-quality standards, but, arguably, can function as one step to achieve them. Substantial improvement necessitates to move beyond the scope of audits, engage company employees in the process of flaw identification, mitigate deficiencies, repair imperfections, and, ultimately, achieve and potentially exceed the company's performance goals.

\section{CONCLUSION \& RECOMMENDATIONS}

The results of our research suggested that the documentation of the GSP's operational departments would not gain the prestigious ISAGO title without findings if the company had decided to pursue the same course. However, the fact the company supported this study is an indication that the particular GSP wants to move from mere compliance to substantial improvements. From the five criteria that were assessed, only the criterion of Controlled Document was compliant with the requirements of ISAGO across all six departments.

The main causes that were withholding the GSP from achieving zero findings in the documentation were:

- The access to the intranet was poor or several employees had no access to the intranet. 
- Personnel was often operating under time pressure which had resulted in the negligence of the documentation.

- Some operational employees were not aware of the available documentation within the operating environment.

- The documentation on the intranet was not effective due to language barriers as multiple nationalities were operating within the GSP.

- The documentation was often not understood or used because the documents contained too much text. This did not apply to one department that had a variety of visuals with appropriate textual explanations.

- $\quad$ Documents were copied from the IGOM $6^{\text {th }}$ edition and AHM $37^{\text {th }}$ edition.

- Most of the departments did not have proof that documentation was received or distributed towards personnel.

- Several GOSARPs were not accurately described in the documentation or were missing in the current documentation.

As the researched GSP is willing to live up to their values and have real conformance rather than the absence of findings, the recognition of the flaws that were identified in this research could be the first step towards a desired organisational behaviour. From this point forward, the GSP must act within all disciplines of the organisation. The researched GSP and other companies with the same goals to establish real conformance should evaluate the points made in this research and reflect them to their own operational environment. However, the conclusions of the criteria will differ per company, as companies tend to have different cultures, operations and the like. In addition, the criteria to measure the current state of documentation but do not measure the involvement of the organisation, commitment throughout the company, and resources available to actually reach true compliance. Therefore, more research needs to be done on this topic or the subjects mentioned previously.

This research revealed that all operational departments had some work to do to achieve zero findings in the next ISAGO audit. The operational departments needed to close the gap between their current state and the preferred zero finding state. Therefore, the current procedures which lack in GOSARP compliance, as well as the copied parts of IATA's guidance materials, should be corrected according to the ISAGO Standards Manual and the GSP's own operational behaviours. After the corrections, the GSP could identify which procedures to be presented in a format that could be understood by all relevant personnel. Based on the preferences of the personnel and the results of previous studies, it could be effective to translate the current procedures into audio-visual based instructions to enhance the transfer of knowledge.

However, it was recommended that the management should collaborate with the personnel that is actually using the documentation. This way, the personnel would experience some kind of ownership on the process which could render the documents understandable by all disciplines, cultures, languages etc. Then, the documentation should be made accessible to all relevant personnel via intranet or another platform. This could be achieved by providing all relevant personnel login data and respective training to navigate through the platform. Another solution could be having hard-copy manuals in the operating environment that could be accessed at all times as well as notifying personnel where and how they could access procedures. Introducing hard-copy manuals does have a negative side note. As procedures change over time, the manuals must be reprinted which could cost money as well as being not environmentally friendly. At last, a procedure of the distribution process should be made by appropriate personnel to ensure that personnel receive, read and understand document revisions. To accomplish this, the operational departments should implement a read and sign book in the department and notify their personnel accordingly. 


\section{REFERENCES}

Amare, G. (2012). U.S. National Library of Medicine. Retrieved from Reviewing the Values of a Standard Operating Procedure: https://www.ncbi.nlm.nih.gov/pmc/articles/PMC3511899/

Barbé B, Verdonck K, Mukendi D, Lejon V, Lilo Kalo JR, et al. (2016) The Art of Writing and Implementing Standard Operating Procedures (SOPs) for Laboratories in LowResource Settings: Review of Guidelines and Best Practices. PLOS Neglected Tropical Diseases 10(11): e0005053. https://doi.org/10.1371/journal.pntd.0005053

CANSO. (2011). Improving Business Performance through Auditing: A CANSO Standard of Excellence. CANSO, 7-16.

Henman, M. (2017, 2 22). From Conflict to Cooperation: Building Stronger Cross Cultural Teams. Retrieved from Business.com: https://www.business.com/articles/from-conflictto-cooperation-building-stronger-cross-cultural-teams/

IATA. (2017). ISAGO Standards Manual: Effective September 2017 (Report No. 6). Retrieved from https://www.aviation-quality-services.com/fileadmin/files/GOSM_6thEdition-Sept2017.pdf

IATA. (2017) Airport Handling Manual (AHM) 37th edition Effective 1 January 2017 - 31 December. Montreal: International Air Transport Association.

IATA. (2017) IATA Ground Operations Manual (IGOM) 6 $6^{\text {th }}$ edition Effective 1 January 2017 31 December. Montreal: International Air Transport Association.

ICAO. (2016, 08 16). Agenda Item 33: Aviation safety and air navigation monitoring and analysis.

Retrieved

from

icao.int: https://www.icao.int/Meetings/a39/Documents/WP/wp_126_en.pdf

Beluško, M., Hegedüš, M., and Fedorko, G. (2016). Creating Visual Work Instructions to Ensure Safe and Fluent Operation of the Semi-automatic Production Lines. Retrieved from degruyter: https://www.degruyter.com/downloadpdf/j/eng.2016.6.issue-1/eng2016-0097/eng-2016-0097.pdf

Peterson, D. C. (2006). Assuring the Effective Use of Standard Operating Procedures (SOPs) In Today's Workforce. Retrieved from Biopharminternational: http://home.sandiego.edu/ josephprovost/Assuring $\% 20$ the $\% 20$ Effective $\% 20$ Use $\% 20$ of $\% 20$ Standard $\% 20$ Operating $\% 20$ Procedures $\% 20$ (SOPs) $\% 20 \ln \% 20$ Today $\% 27 \mathrm{~s} \% 20 \mathrm{~W}$ orkforce\%20-\%20SOPs\%20are\%20critical\%20to\%20efficient\%20operat.pdf

Schiesser, R. (2002). Evaluating System Management Process Documentation. Retrieved from harriskern: http://www.harriskern.com/wp-content/uploads/2012/05/EvaluatingSystem-Management-Process-Documentation.pdf

Suidan, J. (2010, March 16). ISAGO IATA Safety Audit for Ground Operations - ppt download. Retrieved from http://slideplayer.com/slide/3543441/ 